\title{
Caseinolytic protease P (CLPP) activated by ONC201 inhibits proliferation and promotes apoptosis in human epithelial ovarian cancer cells by inducing mitochondrial dysfunction
}

\author{
Xinxin Kou, Hui Ding, Lei Li, Hongtu Chao \\ Department of Gynaecology, Cancer Hospital Affiliated to Zhengzhou University, Zhengzhou, China \\ Contributions: (I) Conception and design: X Kou, H Chao; (II) Administrative support: H. Chao; (III) Provision of study materials or patients: H \\ Ding, L Li; (IV) Collection and assembly of data: X Kou, H Ding, L Li; (V) Data analysis and interpretation: X Kou, H Ding; (VI) Manuscript \\ writing: All authors; (VII) Final approval of manuscript: All authors. \\ Correspondence to: Prof. Hongtu Chao, PhD. Department of Gynaecology, Cancer Hospital Affiliated to Zhengzhou University, No. 127, Dongming \\ Road, Zhengzhou 450008, China. Email: zlyychaohongtu1138@zzu.edu.cn.
}

Background: Caseinolytic protease $\mathrm{P}$ (CLPP) is a mitochondrial specific protein which has been reported to be related to tumor cell apoptosis. This study aims to explore the roles of CLPP in human epithelial ovarian cancer (EOC).

Methods: We determined CLPP expression in paracancerous tissues and cancer tissues obtained from 20 EOC patients, and also in 4 EOC cell lines, and one normal ovarian cell line (IOSE-80). We knocked CLPP expression down in SK-OV-3 and A2780 cells and overexpressed it in SW626 and OVcar3 cells. The effect of CLPP expression on cell proliferation, mitochondrial membrane potential, and apoptosis was then assessed by flow cytometry assay. Furthermore, the effect of ONC201 (agonist of CLPP) on the EOC cell lines was also investigated.

Results: The CLPP expression was markedly down-regulated in EOC cancer tissues, and the Kaplan-Meier Plotter database revealed its low expression was linked to poor prognosis in EOC patients. Low expression of CLPP up-regulated the expression of NADH: ubiquinone oxidoreductase subunit A12 (NDUFA12), succinate dehydrogenase complex flavoprotein subunit A (SDHA), and succinate dehydrogenase complex iron sulfur subunit B (SDHB), which are key members of the mitochondrial respiratory chain, and these up-regulated proteins further led to the increase of mitochondrial membrane potential, cell proliferation promotion and neoplasm metastasis. Conversely, while overexpression of CLPP led to the opposite results, including inducing the decrease of mitochondrial membrane potential and apoptosis. In addition, stimulation with ONC201 enhanced the function of CLPP in SW626 and OVcar3 cells, and silencing of CLPP could neutralize the effect of ONC201.

Conclusions: Our findings suggest that CLPP mediated mitochondrial dysfunction inhibits the proliferation and migration of EOC cells.

Keywords: Epithelial ovarian cancer (EOC); caseinolytic protease P (CLPP); mitochondria; cell apoptosis

Submitted Jul 30, 2021. Accepted for publication Sep 09, 2021.

doi: $10.21037 / \mathrm{atm}-21-4321$

View this article at: https://dx.doi.org/10.21037/atm-21-4321 


\section{Introduction}

Epithelial ovarian cancer (EOC) is a common gynecological malignant tumor, accounting for $50-70 \%$ of ovarian tumors (1). Nearly 230,000 women are diagnosed and 150,000 die of the disease annually worldwide (2), and the trend in both is increasing $(3,4)$. More than $70 \%$ of patients are diagnosed with advanced-stage EOC and widespread metastasis because of its asymptomatic nature (5). In addition, a very low overall 5-year survival is seen due to high recurrence rates resulting from chemoresistance $(6,7)$. Multimodal treatment, including cytoreduction followed by platinum- and taxane-based chemotherapy, is the mainstay of initial treatment (8). Although researchers have made considerable efforts to improve early diagnosis and treatment, most patients are chemotherapy resistance, and tumor metastasis and recurrence are still the main challenges of clinical treatment $(9,10)$. In advanced stages, extrapelvic planting, retroperitoneal or inguinal lymph node metastasis, and superficial liver metastasis are always seen $(11,12)$. Therefore, it is important to find more drug targets to provide new treatment schemes allowing patients to resist chemoresistance. Enhancing aerobic respiration may be one of chemoresistance mechanisms. Upregulated aerobic respiration may produce more energy to deliver drugs out of cancer cells to promote cells survival. Therefore, targeting to mitochondrion might be a good drug therapy strategy.

Aerobic respiration chiefly occurs in the mitochondrion, and studies on its role in cancer suggest targeting it may be a new way to treat EOC (13). Mitochondria have their own protein synthesis and degradation apparatus to maintain normal functions, and mitochondrial caseinolytic protease $\mathrm{P}$ (CLPP) is one of the 15 mitochondrial proteases. CLPP is an oligomeric serine protease in the mitochondrial matrix (14) that is structurally similar to the cytoplasmic/ nuclear proteasome, which has been found to be a potential target for cancer therapy (15). It has a doubleringed tetradecameric structure with a hollow chamber containing proteolytic active sites, and its main function in mitochondria is to degrade misfolded proteins $(16,17)$. CLPP has been found to be related to tumor cell death and apoptosis $(18,19)$. As an anti-cancer compound, ONC201 has been demonstrated to inhibit multiple cancer cell lines with low toxicity (20), and researchers have shown that it can bind CLPP non-covalently to induce mitochondrial dysfunction mediated apoptosis (16). However, whether the expression level of CLPP relates to the indication or prognosis of EOC patients is unclear. Therefore, we investigated the biologic effect and mechanism of CLPP in EOC cells. We present the following article in accordance with the MDAR reporting checklist (available at https:// dx.doi.org/10.21037/atm-21-4321).

\section{Methods}

\section{Patients and sampling}

Para-carcinoma specimens and pathological specimens were collected from 20 EOC patients who did not receive chemotherapy after operation, and the histopathological diagnosis, stage, and grade of the EOC samples were identified by a pathologist from the Cancer Hospital Affiliated to Zhengzhou University. Each participant knew the purpose and meaning of the experiment and signed a written informed consent. The study protocol was reviewed and approved by the Institutional Review Board of Cancer Hospital Affiliated to Zhengzhou University (No. 20191016), in accordance with the Declaration of Helsinki (as revised in 2013), and informed consent was taken from all the patients.

\section{Cell culture and treatment}

Four human EOC cell lines (SW626, SK-OV-3, A2780 and OVcar3) and one normal ovarian cell line (IOSE-80) were obtained from the Institute of Biochemistry and Cell Biology, Chinese Academy of Sciences. The five cell lines were cultured with Dulbecco Minimum Essential Medium (DMEM) (Thermo Fisher Scientific, Waltham, MA, USA) supplemented with fetal bovine serum (FBS, 10\%) (Gibco-BRL, USA), $100 \mu \mathrm{g} / \mathrm{mL}$ streptomycin, and $100 \mu \mathrm{g} / \mathrm{mL}$ penicillin G (Beyotime, Shanghai, China) at $37{ }^{\circ} \mathrm{C}$ in a humidified atmosphere containing $5 \% \mathrm{CO}_{2}$. To knock down the expression of CLPP in SK-OV-3 and A2780, specific siRNAs targeted to CLPP were synthesized by GenePharma (Shanghai, China), and to overexpress CLPP in SW626 and OVcar3, CLPP CDS was cloned and recombined into the pGL3.0-basic plasmid. Recombinant plasmid or siRNAs were transfected into cells through Lipofectamine 2000 Reagent (Invitrogen, Carlsbad, CA, USA) according to the manufacturer's instructions.

\section{Real time quantitative PCR}

RNA was extracted by TRIzol reagent (Invitrogen) and the 
cDNA was generated with RNA ( $1 \mu \mathrm{g})$, random primers and Primescript reverse transcriptase (Takara, Japan). The primers were designed through the Primer Premier 6.0 software. The primer sequences used for CLPP were 5'-ACTCAGTCATTCAACAGATGGC-3' (forward) and 5'-CCCTGTCTGCTACACTCCCT-3' (reverse), and for glyceraldehyde 3-phosphate dehydrogenase (GAPDH) was 5'-TGTTCGTCATGGGTGTGAAC-3' (forward) and 5'-ATGGCATGGACTGTGGTCAT-3' (reverse). Quantitative reverse transcription-PCR (QPCR) was carried out in a 7500 Real-Time PCR System (Applied Biosystems, Life Technologies, USA) according to the manufacturer's instructions of the SYBR Green qPCR kit (TOYOBO, Osaka, Japan). The PCR cycling conditions were $1 \mathrm{~min}$ at $94^{\circ} \mathrm{C}, 35$ cycles of denaturing for $30 \mathrm{~s}$ at $94{ }^{\circ} \mathrm{C}$, annealing for $2 \mathrm{~min}$ at $60^{\circ} \mathrm{C}$, and extension for $1 \mathrm{~min}$ at $72{ }^{\circ} \mathrm{C}$, finally saved briefly $5 \mathrm{~min}$ at $72{ }^{\circ} \mathrm{C}$. GAPDH was used as an internal reference gene, and the $2^{-\Delta \Delta \mathrm{Ct}}$ method was applied to analysis gene expression level.

\section{Western blotting}

Total protein was separated from cells and tissue samples through RIPA buffer (protease inhibitor contained) for $0.5 \mathrm{~h}$ on ice then centrifuged, and the BCA Protein Assay kit (Beyotime) was used to access the concentrations of extracted protein. The extracted protein $(20 \mu \mathrm{g})$ was then loaded into a sodium dodecyl sulfate-polyacrylamide gel electrophoresis (SDS-PAGE) under reducing conditions for separation and transferred to a polyvinylidene fluoride membrane, followed by blocking with phosphate-buffered saline with Tween detergent (PBST) containing 5\% evaporated skimmed milk at room temperature for $1 \mathrm{~h}$. After washing three times with PBST, the membranes were immerged with solution containing anti-CLPP (1:800 diluted; Abcam), anti-GAPDH (1:2,000 diluted; Abcam), anti-p-NDUFA12 (1:400 diluted; Abcam), antiSDHA (1:500 diluted; Abcam), anti-SDHB (1:400 diluted; Abcam), anti-CASP3 (1:1,500 diluted; Abcam), or antiBcl-2 (1:1,500 diluted; Abcam) at $4{ }^{\circ} \mathrm{C}$ overnight. The membranes were then washed three times and incubated with HRP labeled anti-IgG antibody (BOSTER, Wuhan, China) at room temperature for $1 \mathrm{~h}$. Finally, the signal was revealed with ECL-Plus reagent (Millipore, Billerica, MA, USA) and exposed to film. The gray value of protein bands were quantified using Image pro plus 6.0 software (Media Cybernetics, MA, USA).

\section{Immunofluorescence}

After treatment, five cell lines after treatment were harvested and fixed with $4 \%$ paraformaldehyde (PFA) $-0.0075 \%$ glutaraldehyde for $15 \mathrm{~min}$, permeabilized with PBST containing $0.1 \%$ Triton $\mathrm{X}-100$, and blocked with $10 \%$ bovine serum albumin (BSA) (Sigma-Aldrich) for $30 \mathrm{~min}$ at room temperature. After incubation with anti-mouse CLPP diluted at $1 / 500$ in PBST for $2 \mathrm{~h}$ at $4{ }^{\circ} \mathrm{C}$, the cells were washed three times with PBST at room temperature, followed by incubation with Alexa FluorR 488 conjugate [F(ab')2-Goat anti-Mouse IgG (H+L) Cross-Adsorbed Secondary Antibody, Life technologies, refA11017] for $1 \mathrm{~h}$. Furthermore, 4',6-diamidino-2-phenylindole (DAPI) was used to stain the nucleus. Finally, images were obtained by a fluorescence microscope (Axio Imager.M2, Zeiss).

\section{Cell viability assay}

Cell viability was measured according to the instructions of the Cell Counting Kit- 8 (Dojindo, Japan). Briefly, $1 \times 10^{4}$ corresponding cells were transferred into 96 -well plates at $12,24,36$, and $48 \mathrm{~h}$ after transfection. Each group had three replicates. Following this, $100 \mu \mathrm{L}$ of solution containing WST-8 was added into each well and placed into an incubator for $4 \mathrm{~h}$ at $37{ }^{\circ} \mathrm{C}$ with $5 \% \mathrm{CO}_{2}$. Subsequently, this was detected at $450 \mathrm{~nm}$ wavelength and the optical density value of each well was recorded by a Microplate Reader (Thermo Scientific).

\section{Flow cytometry}

The Annexin V-FITC/PI Apoptosis Kit (MultiSciences, Hangzhou, China) and Mitochondrial membrane potential test kit (JC-1) (Beyotime) were applied to determine cell apoptosis, cell cycle, and mitochondrial membrane potential through flow cytometry assay, respectively. Cells $\left(5 \times 10^{5}\right)$ were then harvested and washed three times by PBS after transfection, and incubated in $200 \mu \mathrm{L}$ of Annexin V-FITC/PI solution for $15 \mathrm{~min}$ in the dark. Finally, the FITC fluorescence and PI fluorescence was detected by BD FACS Calibur ${ }^{\mathrm{TM}}$ flow cytometry (BD Biosciences, Franklin, NJ, USA).

\section{Wound healing assay}

For the detection of cell migration, corresponding cells 
were placed into six well plates $\left(6 \times 10^{4}\right.$ cells $)$ with preinstalled silicone inserts, then cultured to confluent and pretreated with an artificial wound scratched by a sterile pipette tip. Six randomly selected fields of the wound were recorded by a CX41 Microscopy (Olympus, Tokyo, Japan).

\section{Transwell assay}

Cell metastatic ability was assessed using a Transwell system (Corning, NY, USA). Cells were incubated $36 \mathrm{~h}$ after transfection, then, treated with serum-free medium for $12 \mathrm{~h}$. The medium was then removed and the cells were washed with PBS three times. Next, $700 \mu \mathrm{L}$ medium containing $10 \% \mathrm{FBS}$ was added to the lower chamber and $2 \times 10^{4}$ cells were seeded to the upper chamber, then incubated in air with $5 \% \mathrm{CO}_{2}$ at $37{ }^{\circ} \mathrm{C}$ for $48 \mathrm{~h}$. Subsequently, the cells on the lower side of the filter were fixed with $4 \%$ paraformaldehyde and stained with $0.1 \%$ crystal violet solution. Finally, six randomly selected fields were photographed by a CX41 Microscopy (Olympus) and the cell numbers counted. Cell invasions were similarly accessed as above, excepting a matrigel insert paved between the upper and low chamber.

\section{Statistical analysis}

GraphPad Prism 8 (GraphPad Software, San Diego, CA, USA) and SPSS 20.0 (SPSS, Chicago, IL, USA) were used for visualization and statistical analysis, respectively. All statistical values are showed as means \pm standard deviation (SD), and statistical significance was considered when $\mathrm{P} \leq 0.05$.

\section{Results}

\section{CLPP is lowly expressed in EOC}

To examine the difference in expression of CLPP between para-carcinoma specimens and pathological specimens, QPCR assay and Western blotting assay were applied using tissues from patients with EOC. The results showed the mRNA and protein level of CLPP were both evidently down-regulated in pathological specimens (Figure 1A,1B), and the Kaplan-Meier Plotter database (https://kmplot. com/analysis/index.php? $\mathrm{p}=$ service\&cancer $=$ ovar) revealed that EOC patients with a lower expression of CLPP had a worse prognosis than those with high expression (Figure 1C). Next, we performed RT-qPCR, western blot, and immunofluorescence assays to examine the levels of CLPP expression in the EOC cell lines and normal ovarian cell line. Our results showed that CLPP expression was significantly downregulated in all the EOC cell lines when compared with the IOSE-80 cell line (Figure 2A-2C). These results indicated that CLPP expression was downregulated in EOC.

\section{Silencing of CLPP promotes proliferation, migration, and invasion in SK-OV-3 and A2780 cells}

To investigate whether CLPP affected human ovarian cancer cell proliferation, migration, and invasion, we knocked down its expression in SK-OV-3 and A2780 cells using siRNA. Subsequently, QPCR and Western blotting was applied to test the silencing efficacy, while CCK8 assay and EdU staining were used to assess cell viability. The data obtained showed that the expression of CLPP was observably decreased (Figure 3A). Consistently, Western blotting showed that three mitochondrial respiratory chain related proteins, NDUFA12, SDHA, and SDHB, were up-regulated after transfection, which demonstrated the mitochondrial respiratory metabolism enhancing of tumor cells (Figure 3B). Moreover, CLPP silencing significantly promoted cell proliferation $36 \mathrm{~h}$ after transfection (Figure 3C-3E). Further, the silencing of CLPP observably inhibited cell apoptosis, while the monomer ratio of JC-1 was significantly decreased, which means the mitochondrial membrane potential was increased (Figure $4 A-4 D$ ). In addition, Transwell assay and wound healing assay revealed that knock-down of CLPP significantly promoted cell migration and invasion (Figure 4E-4H).

\section{Overexpression of CLPP inhibits mitochondrial function and induces apoptosis in SW626 and OVcar3 cells}

To further prove the function of CLPP in EOC cells, we overexpressed CLPP in SW626 and OVcar3 cells. The QPCR and Western blotting assay showed that the expression of CLPP was observably increased (Figure 5A), Further, Western blotting showed that NDUFA12, SDHA, and SDHB were down-regulated after transfection, which demonstrated the mitochondrial respiratory metabolism reducing of tumor cells (Figure $5 B$ ). While CCK8 assay, EdU staining, and flow cytometry showed CLPP overexpression significantly inhibited cell viability and proliferation $36 \mathrm{~h}$ after transfection (Figure $5 C-5 E$ ). Results of flow cytometry assay revealed that the highly 


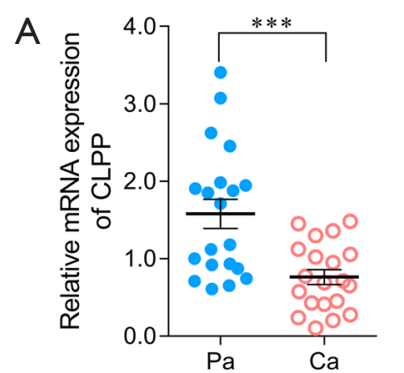

B

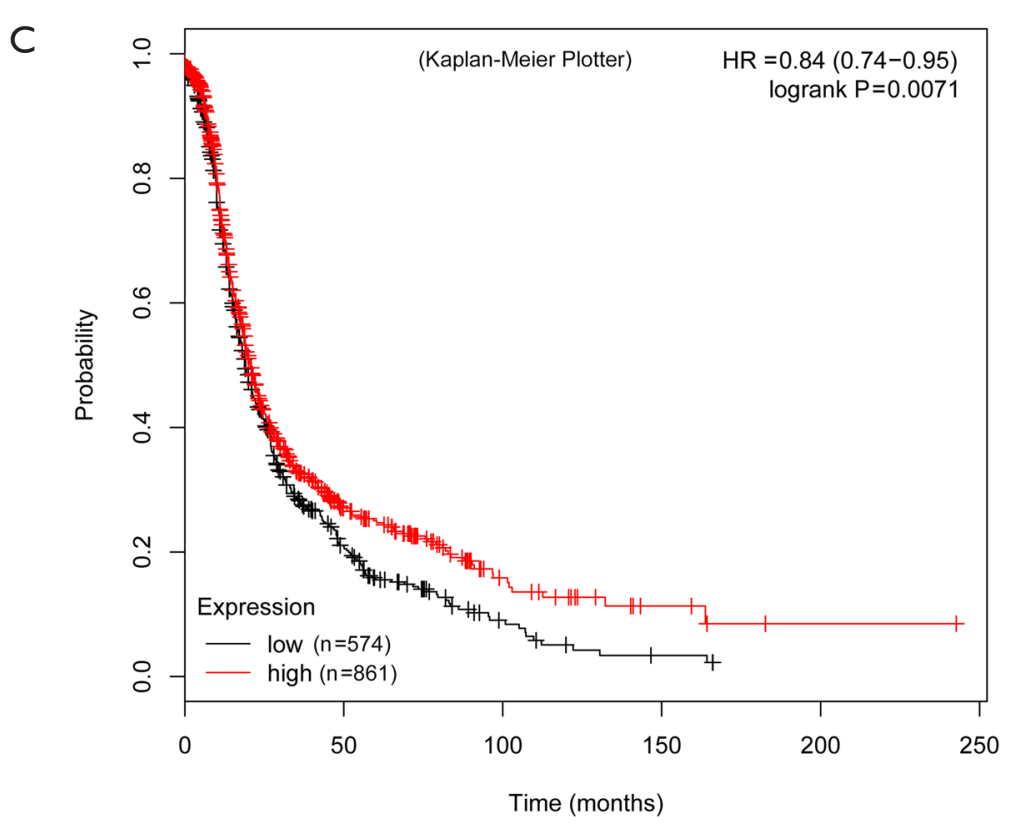

Figure 1 Expression level of caseinolytic protease P (CLPP) in pathological specimens (Ca) and in para-carcinoma specimens (Pa). (A) mRNA level of CLPP detected by QPCR ( $n=20,{ }^{* * *} \mathrm{P}<0.001$, paired $t$-test); (B) protein level of CLPP detected by Western blotting; (C) effect of CLPP expression level on prognosis of EOC patients in the Kaplan-Meier Plotter database.

expressed CLPP evidently promoted cell apoptosis, while the monomer ratio of $\mathrm{JC}-1$ was significantly increased by that, which means the mitochondrial membrane potential was decreased (Figure 6A-6D). In addition, Transwell assay and wound healing assay revealed that expression of CLPP observably suppressed the metastatic ability of EOC cells (Figure 6E-6H).

\section{ONC201, agonist of CLPP, inbibits proliferation, migration, and invasion of EOC cells}

An agonist of CLPP, ONC201 was also applied to investigate the function of CLPP in EOC cells, and SW626 and OVcar3 cells were stimulated with ONC201 $(3 \mu \mathrm{mol} / \mathrm{L})$ only or ONC201 + si-CLPP. The QPCR and Western blotting assay revealed that the expression of CLPP was significantly up-regulated after stimulation with ONC201 only, while significantly down-regulated after transfected with si-CLPP (Figure 7A). Western blotting showed that the protein level of NDUFA12, SDHA, SDHB, and Bcl-2 decreased, while that of CASP3 increased after ONC201 stimulation (Figure 7B). CCK8 assay and EdU staining showed that cell viability and proliferation were significantly inhibited by ONC201, while si-CLPP could reduce the inhibition to some extent (Figure $7 C-7 E$ ). Further, the stimulation of ONC201 observably promoted cell apoptosis, and the monomer ratio of JC-1 was significantly increased (Figure $8 A-8 D$ ). In addition, Transwell assay and wound healing assay revealed that stimulation of ONC201 significantly inhibited cell migration and invasion (Figure 8E-8H). Of note, silencing of CLPP could reduce the effects of ONC201 on EOC cells. 
A

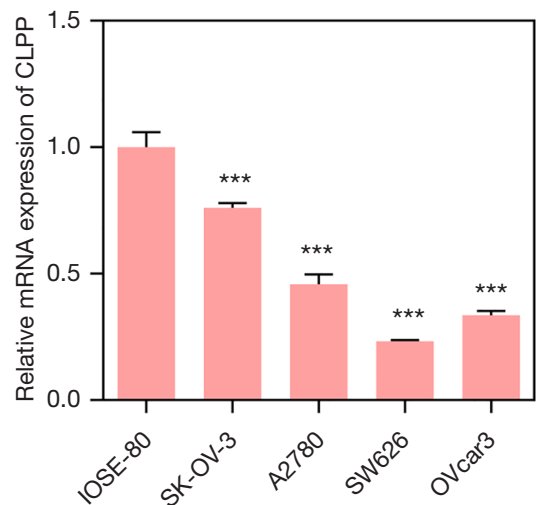

C

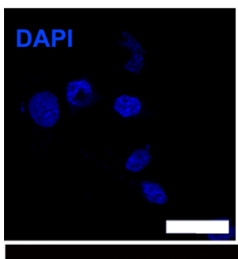

Merge
B

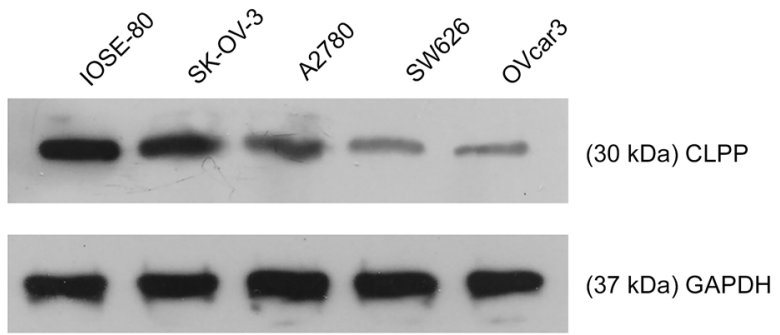

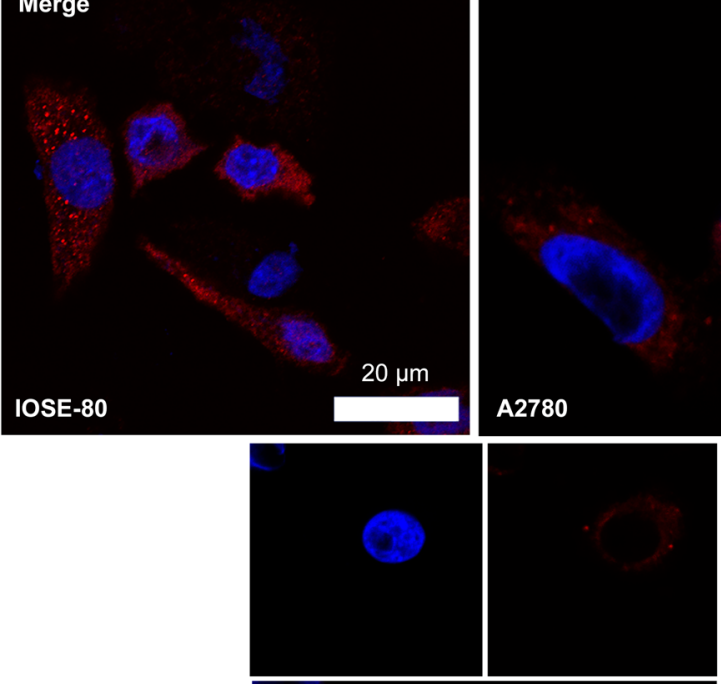
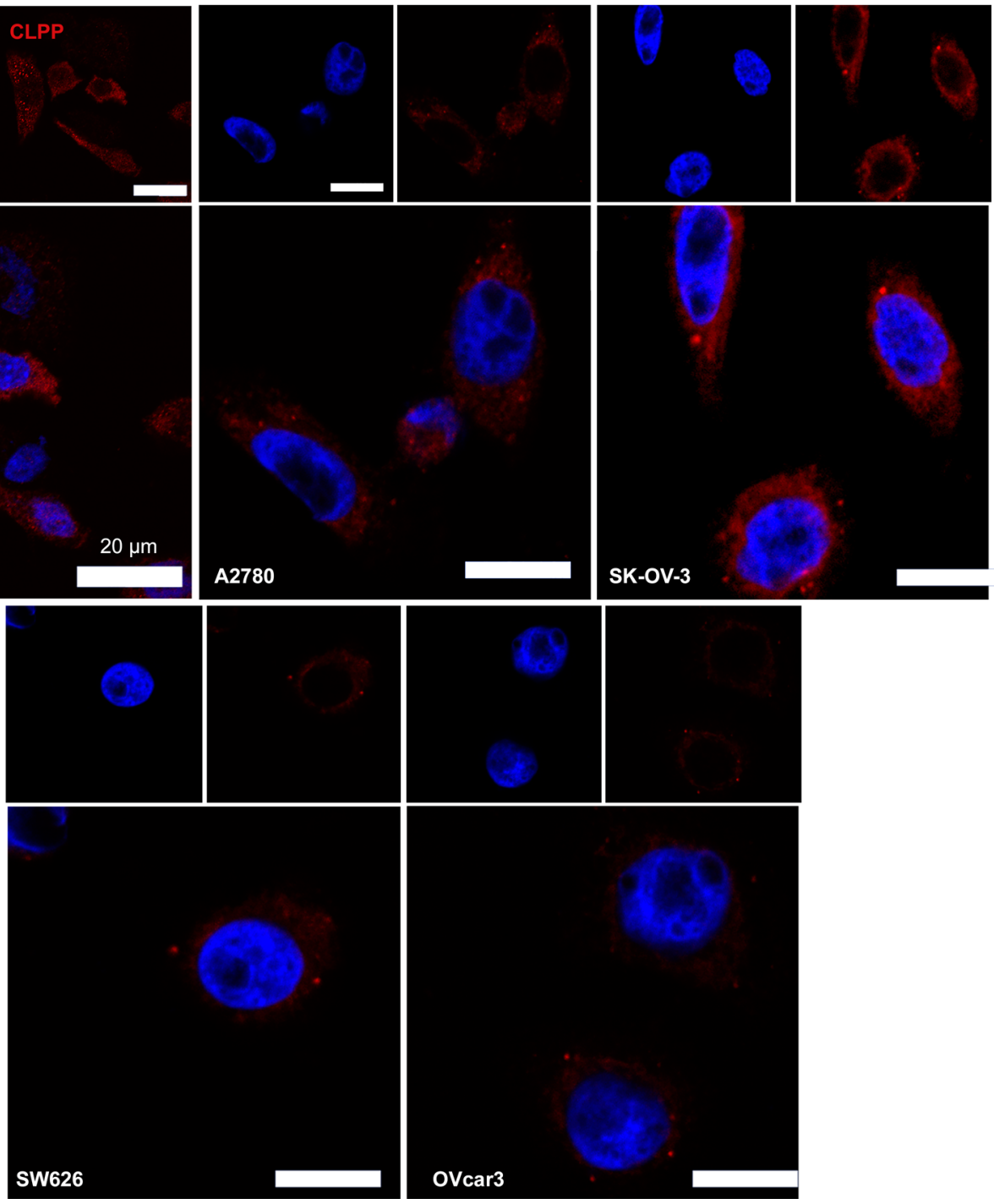

Figure 2 Expression level of caseinolytic protease P (CLPP) in IOSE-8, SK-OV-3, A2780, SW626, and OVcar3 cells. (A) mRNA level of CLPP detected by QPCR ( $\mathrm{n}=3,{ }^{* * *} \mathrm{P}<0.001$, paired $t$-test); (B) protein level of CLPP detected by Western blotting; (C) distribution of CLPP in different cell detected by immunofluorescence. Scar bar $=20 \mu \mathrm{m}$. 
A

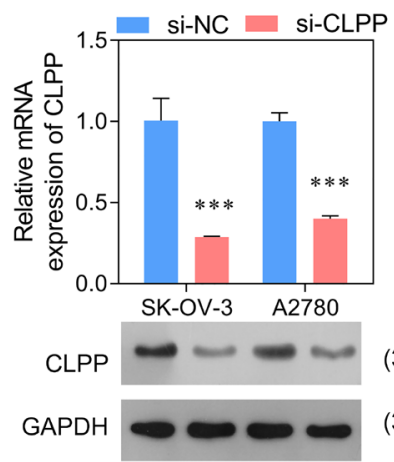

$(30 \mathrm{kDa})$

$(37 \mathrm{kDa})$
B

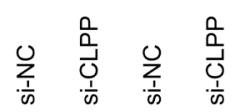

(17 kDa) NDUFA12

(72 kDa) SDHA

(32 kDa) SDHB

(37 kDa) GAPDH
C

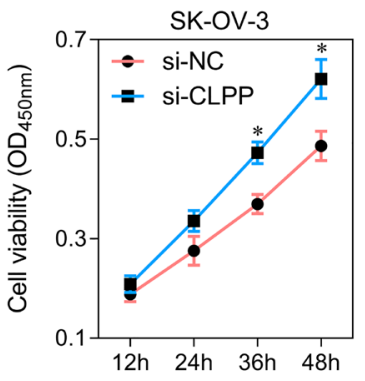

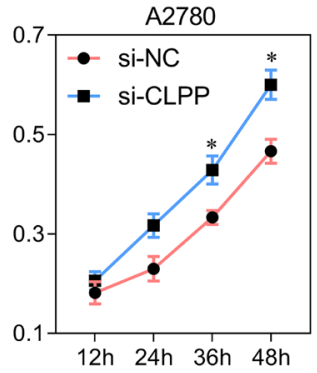

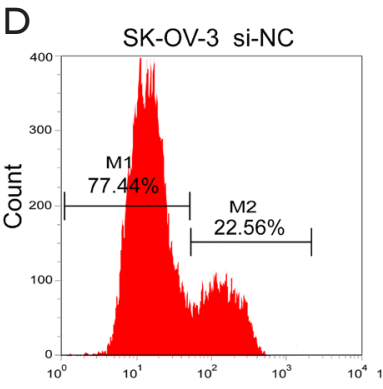

SK-OV-3 si-CLPP

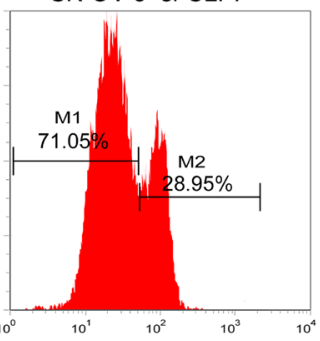

A2780 si-NC

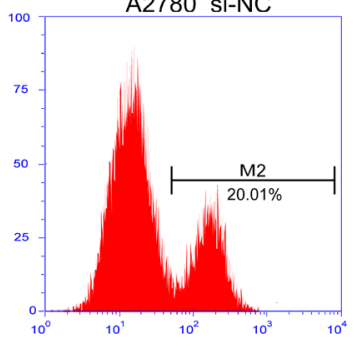

A2780 si-CLPP

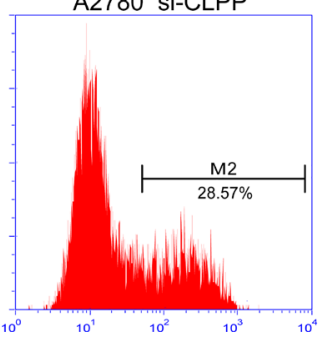

Figure 3 Silencing of caseinolytic protease P (CLPP) promoted the viability and proliferation of SK-OV-3 and A2780 cells. SK-OV-3 and A2780 cells were transfected with siRNA specifically targeting CLPP. (A) Levels of CLPP expression after siRNA transfection were detected by RT-qPCR and Western blotting $\left(\mathrm{n}=3,{ }^{* * *} \mathrm{P}<0.001\right.$, pared $t$-test). (B) Levels of NDUFA12 and SDHA protein expression after siRNA transfection were detected by Western blotting. (C-E) Cell viability at 12, 24, 36, and $48 \mathrm{~h}$ and proliferation at $36 \mathrm{~h}$ after siRNA transfection were measured by the CCK8 assay and Edu staining, respectively $\left({ }^{*} \mathrm{P}<0.05,{ }^{* *} \mathrm{P}<0.01\right.$, paired t-test). 
A
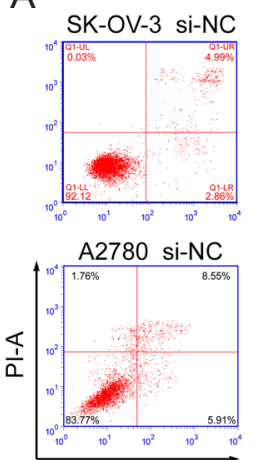

Annexin V-FITC-A

$\mathrm{E}$

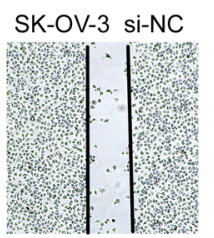

A2780 si-NC

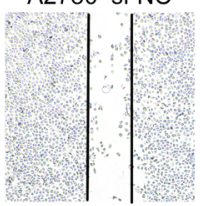

B
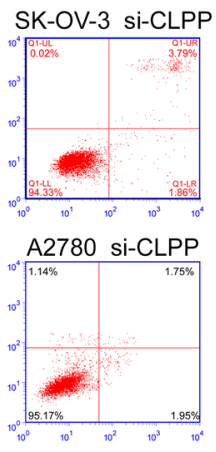

$10^{4} \quad 10^{\circ}$
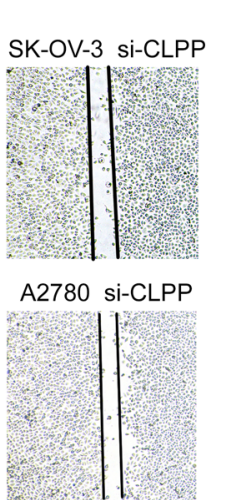

$\mathrm{F}$
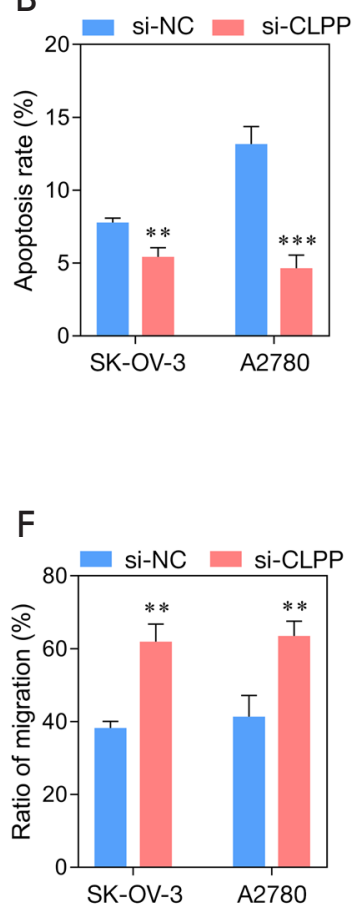

C
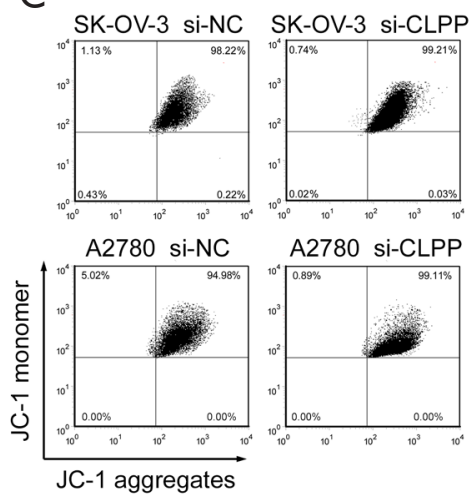

A2780 si-CLPP

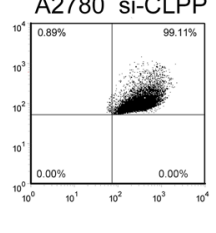

G

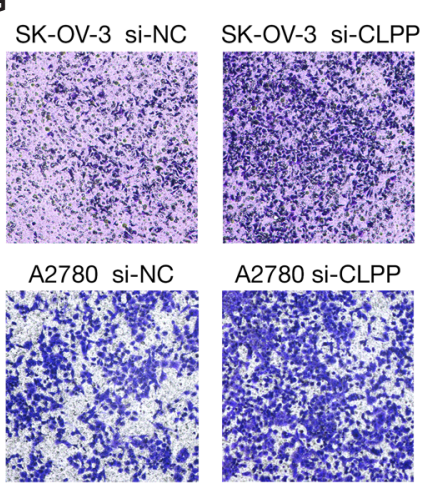

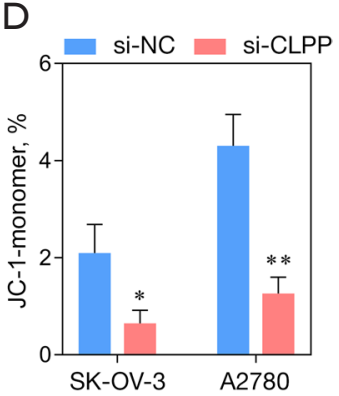

$\mathrm{H}$

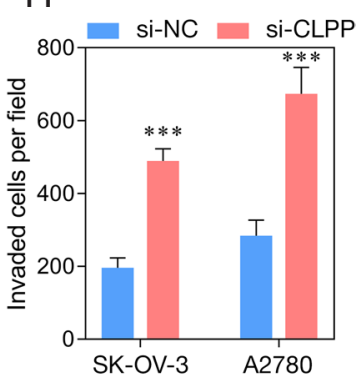

Figure 4 Silencing of caseinolytic protease P (CLPP) inhibited cell apoptosis and promoted cell migration and invasion. (A,B) Flow cytometry assays revealed the rates of the SK-OV-3 and A2780 cell apoptosis at $36 \mathrm{~h}$ after transfection; (C,D) mitochondrial membrane potential was measured by flow cytometry; (E,F) cell migration was detected by the wound healing assay ( $\times 40$ magnification); (G,H) cell invasion was detected by the Transwell assay $\left(\times 100\right.$ magnification). ${ }^{*} \mathrm{P}<0.05,{ }^{* *} \mathrm{P}<0.01,{ }^{* * *} \mathrm{P}<0.001$, paired $t$-test.

\section{Discussion}

Although there have been many efforts to improve treatment, therapy for EOC has remained largely unchanged and ineffective (21), and there remains an urgent need to discover valid drug targets to diagnose and treat EOC patients. Here, we identified CLPP as one of the 15 mitochondrial proteases whose agonist might be cytotoxic to EOC cells, and we testified that the expression of CLPP in four EOC cells lines was significantly lower compared with normal ovarian cell line IOSE-80, which implied us CLPP might be related to tumor progression.

Evidence has revealed that mitochondria play a major role in the maintenance and therapy resistance of leukemias $(16,22)$ and certain solid tumors (23). In addition, studies have found that the effective destruction of mitochondrial integrity may be used as a tumor treatment strategy $(24,25)$.

CLPP is a protease conserved from bacteria to humans and is relevant to the mitochondrial unfolded protein response. CLPP could coordinate fertility, hearing, growth (26), and cancer cell viability $(16,22)$, and its hyperactivation selectively induced cancer cell death through selectively degradating respiratory chain protein substrate and inducing mitochondrial disorder, which is independent of p53 status (16). In the present study, the expression of CLPP was remarkably higher in cancer tissue than in para-carcinoma tissue. Knock-down of CLPP could promote cell proliferation, migration, and invasion in SK-OV-3 and A2780 cells, while overexpression inhibited these processes in SW626 and OVcar3 cells. Proteins interacting with CLPP play an important role in respiratory chain and mitochondrial metabolism (22), and NDUFA12, SDHA, and SDHB are key members of the mitochondrial respiratory chain whose expression was affected by CLPP. ONC201 is an anti-cancer compound that effectively kills a variety of cancer cells while less toxic to normal cells (27-30), 
A

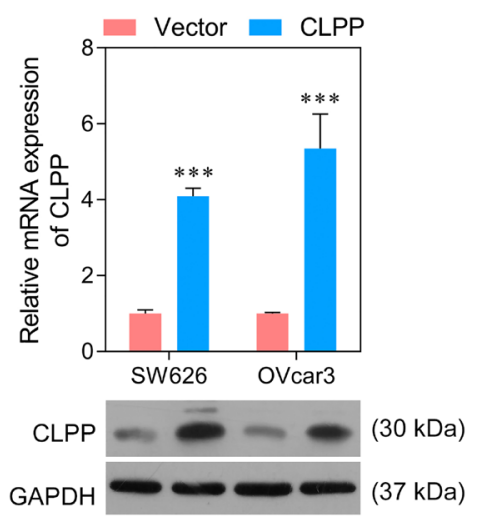

B

$\begin{array}{llll}\frac{1}{0} & 0 & \frac{1}{0} & 0 \\ \text { ग } & 0 & 0 & \frac{0}{0}\end{array}$

$=(17 \mathrm{kDa}) \mathrm{NDUFA} 12$

$-2-(72 \mathrm{kDa}) \mathrm{SDHA}$

$m=2 \mathrm{kDa}) \mathrm{SDHB}$

$-\infty-(37 \mathrm{kDa}) \mathrm{CASP} 3$

$-\infty-(26 \mathrm{kDa}) \mathrm{Bcl}-2$

SW626 OVcar3

C
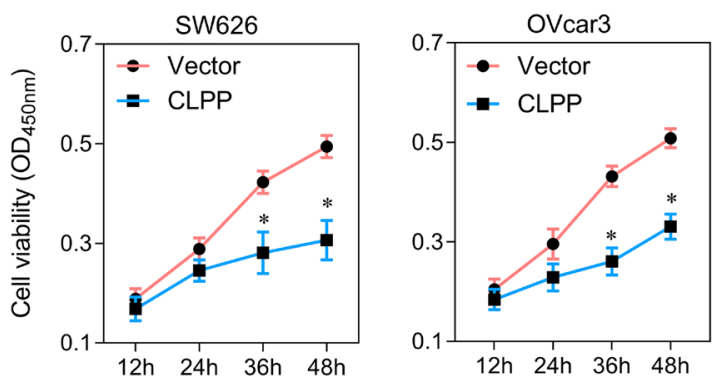

$\mathrm{D}$
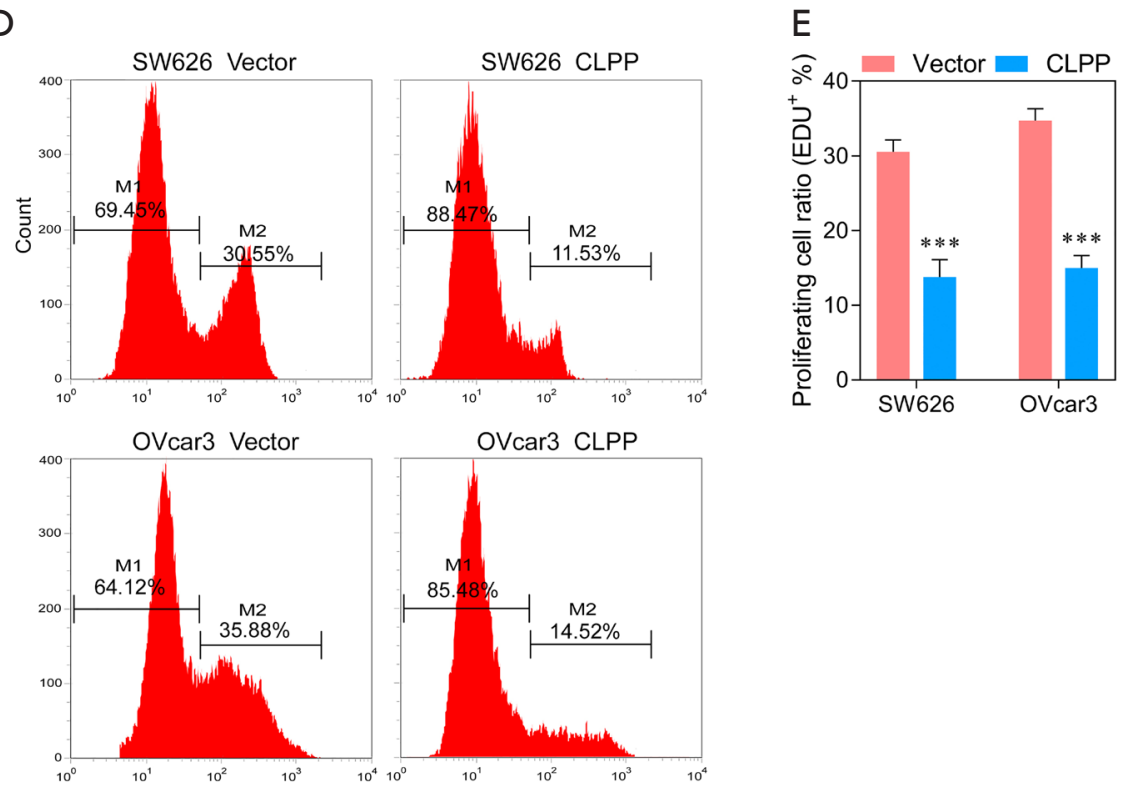

Figure 5 Overexpression of caseinolytic protease P (CLPP) reduced the viability and proliferation of SW626 and OVcar3 cells. SW626 and OVcar3 cells were transfected with the pGL3.0-CLPP plasmid. (A) The levels of CLPP expression after transfection were detected by RT-qPCR and Western blotting $\left(\mathrm{n}=3,{ }^{* * *} \mathrm{P}<0.001\right.$, paired $t$-test); (B) the levels of NDUFA12, SDHA, SDHB, CASP3, and Bcl-2 proteins after siRNA transfection were detected by Western blotting; (C-E) cell viability at 12, 24, 36, and $48 \mathrm{~h}$ and proliferation at $36 \mathrm{~h}$ after siRNA transfection were measured by the CCK8 assay and Edu staining, respectively $\left({ }^{*} \mathrm{P}<0.05,{ }^{* *} \mathrm{P}<0.001\right.$, paired $t$-test). 


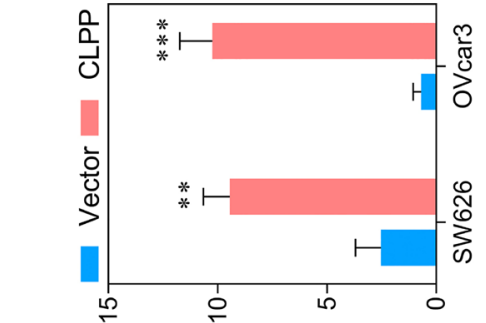

○

(\%) дәшоиоu- L-כr

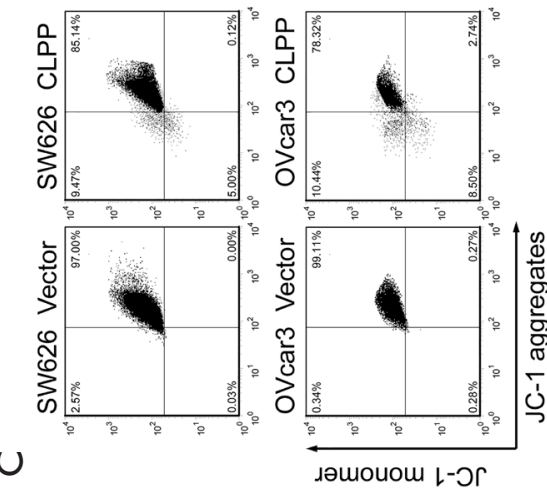

$\infty$

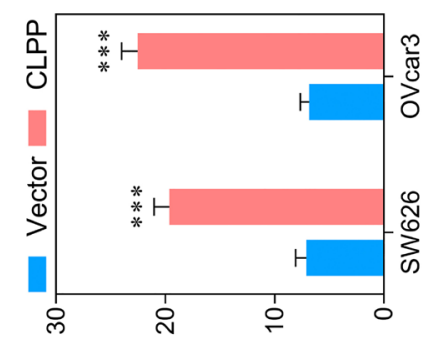

(\%) әəeג s!soldod $\forall$
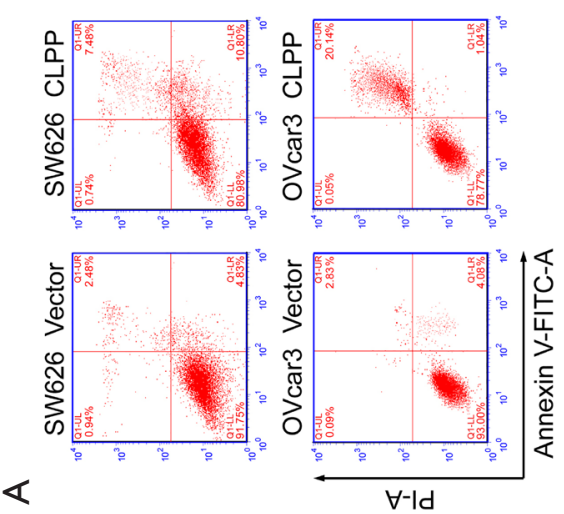

I
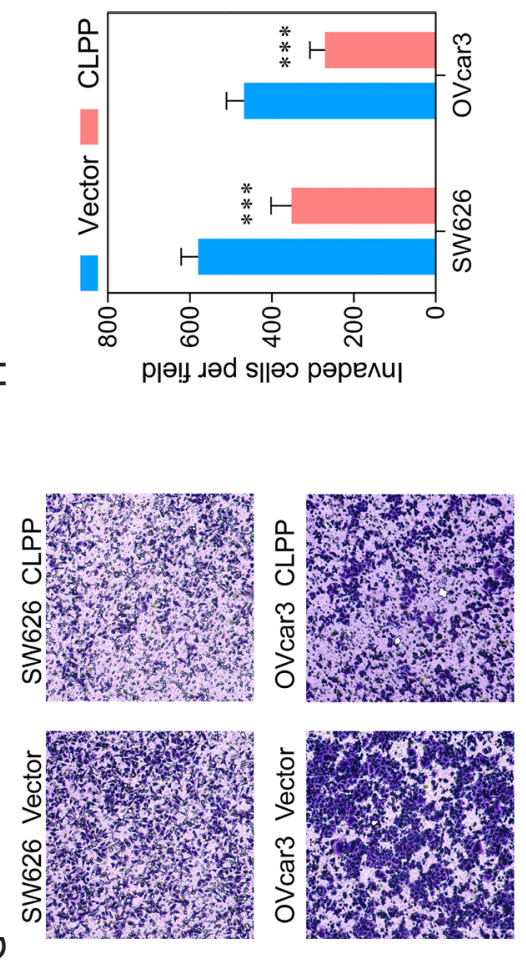

ш

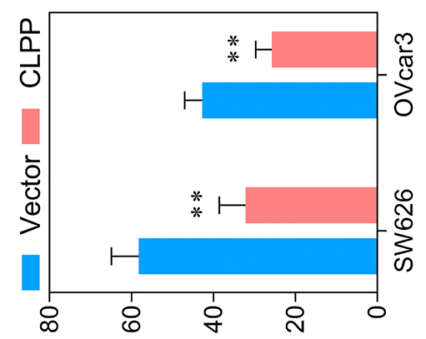

(\%) uo!ıeıbịu fo o!̣ey
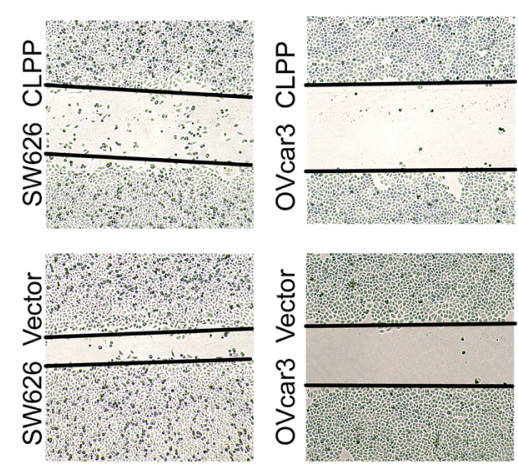

ш

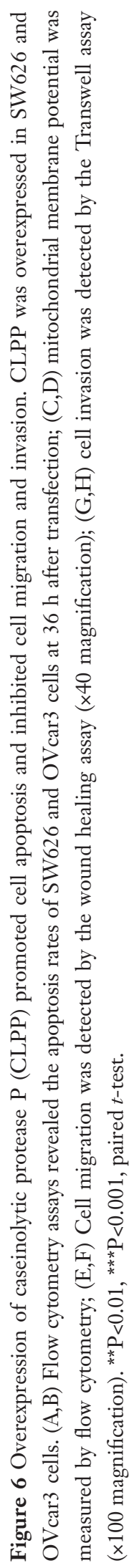



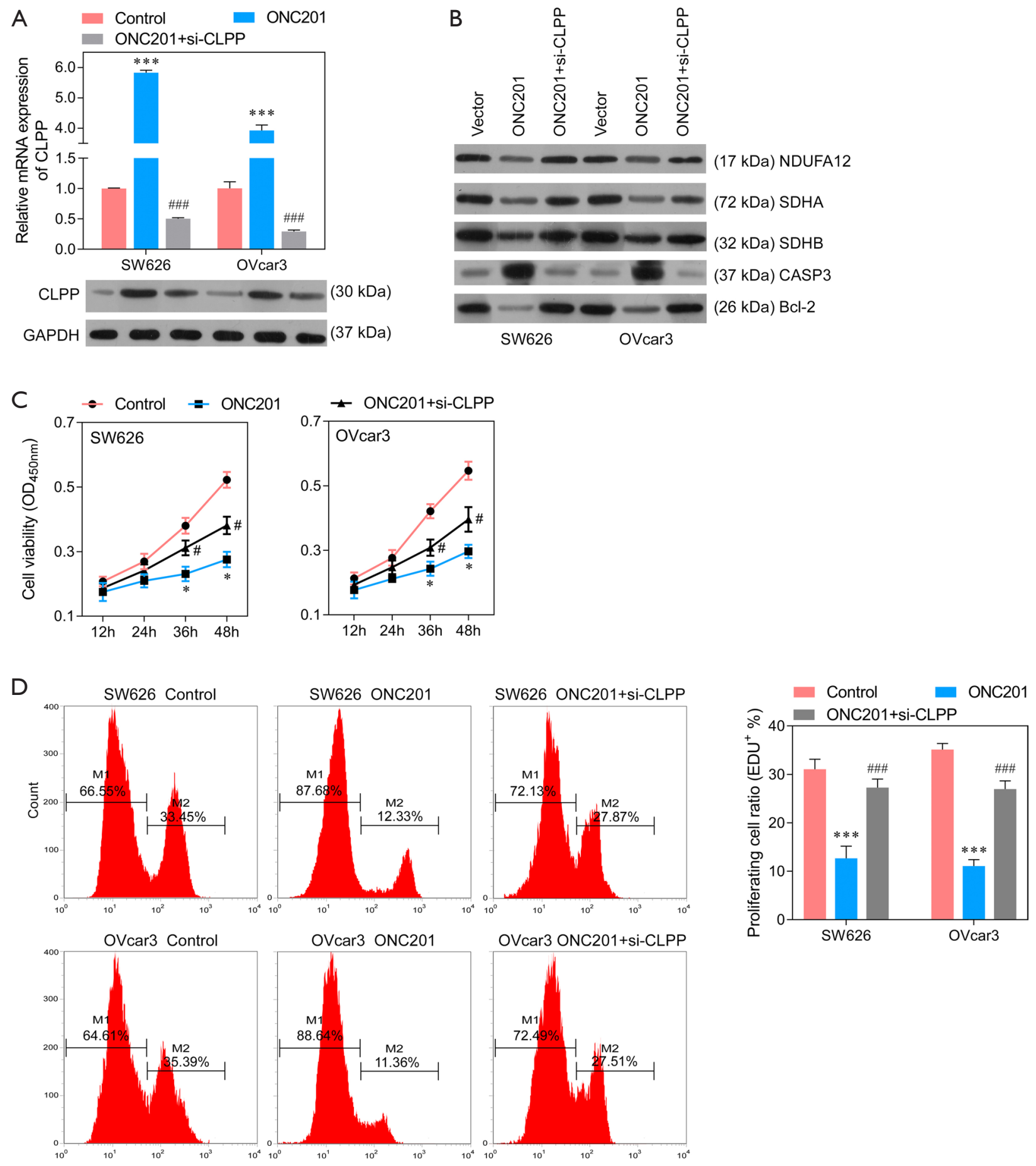

Figure 7 ONC201 reduced the viability and proliferation of SW626 and OVcar3 cells. SW626 and OVcar3 cells were treated with ONC201 after transfection with control siRNA or siRNA targeting caseinolytic protease P (CLPP). (A) Levels of CLPP expression were examined via RT-qPCR and Western blotting ( $\mathrm{n}=3,{ }^{* *} \mathrm{P}<0.001$, One-Way ANOVA). (B) The levels of NDUFA12, SDHA, SDHB, CASP3, and Bcl-2 proteins were detected by Western blotting. (C-E) Cell viability at 12, 24, 36, and $48 \mathrm{~h}$ and proliferation at $36 \mathrm{~h}$ after transfection were measured by the CCK8 assay and Edu staining, respectively. ${ }^{*} \mathrm{P}<0.05,{ }^{* * *} \mathrm{P}<0.001$, vs. Control; ${ }^{\#} \mathrm{P}<0.05$, ${ }^{\# \# \#} \mathrm{P}<0.001, v s$. ONC201, OneWay ANOVA. 
A
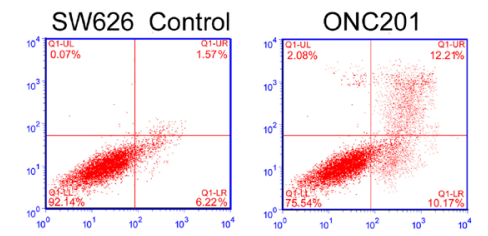

OVcar3 Control
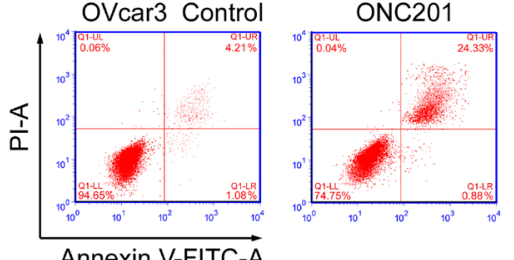

C

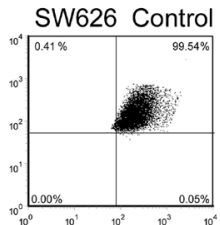

OVcar3 Contro

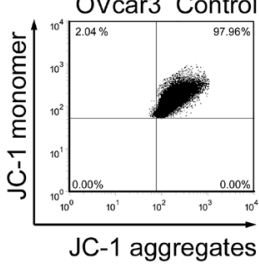

E

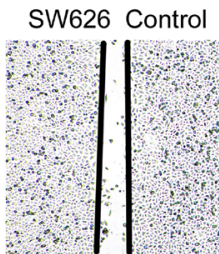

OVcar3 Control
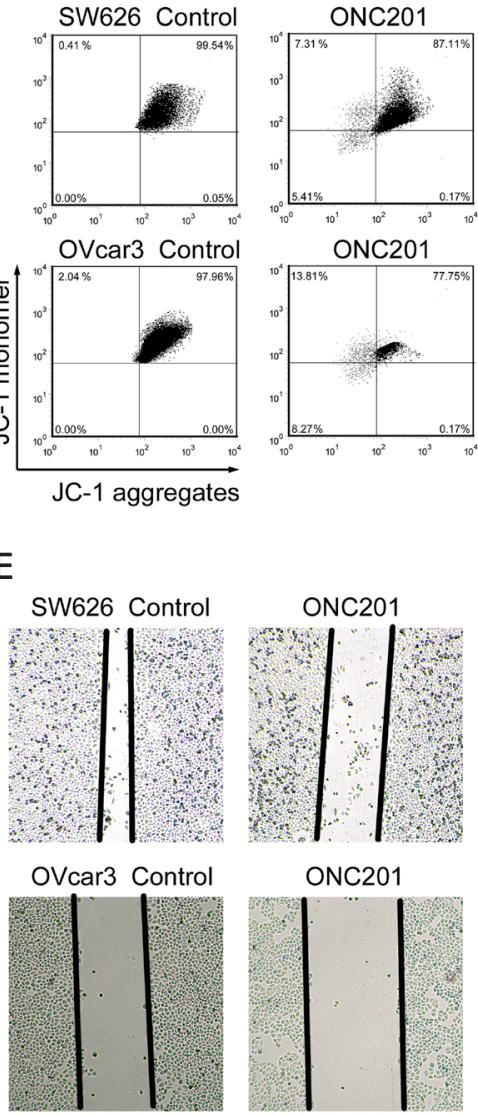

ONC201+si-CLPP
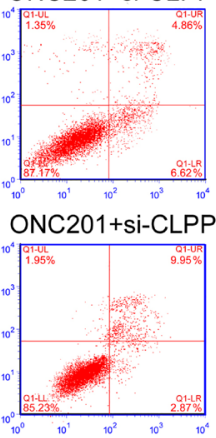

ONC201+si-CLPP

ONC201+si-CLPP

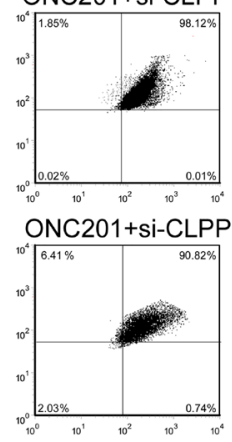

D

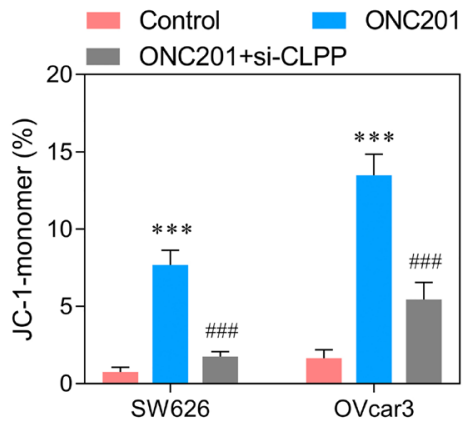

F

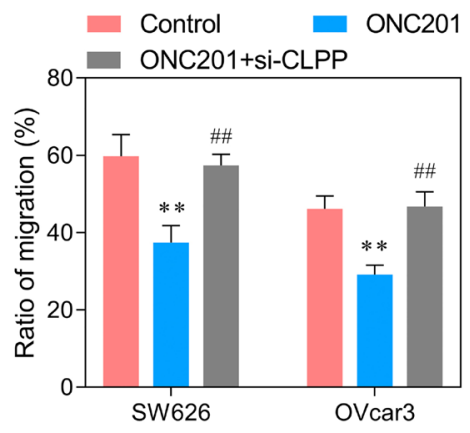


G

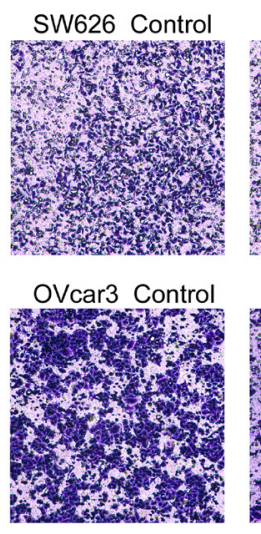

ONC201

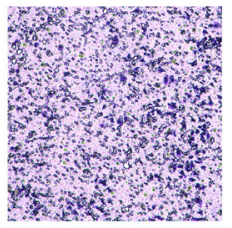

ONC201

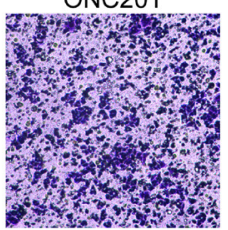

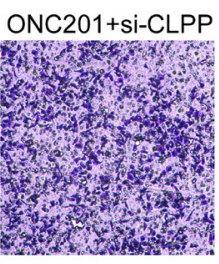

ONC201+si-CLPP

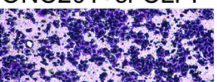

$\mathrm{H}$

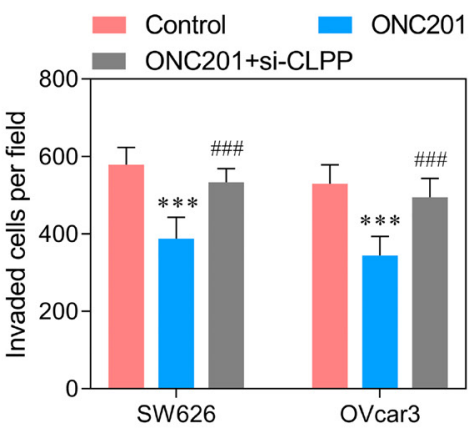

Figure 8 ONC201 promoted apoptosis and inhibited the migration and invasion of SW626 and OVcar3 cells. (A,B) Flow cytometry assays revealed the apoptosis rates of SW626 and OVcar3 cells at $36 \mathrm{~h}$ after treatment with ONC201; (C,D) mitochondrial membrane potential was measured by flow cytometry; (E,F) cell migration was detected by wound healing assay ( $\times 40$ magnification); (G,H) cell invasion was

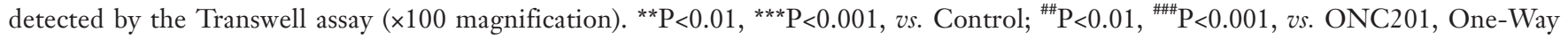
ANOVA.

and Ishizawa et al. confirmed that CLPP was a direct target for ONC201 (16). In EOC cells, ONC201 activated CLPP expression, followed by decrease of NDUFA12, SDHA, and SDHB proteins, and the reduction of these protein could induce mitochondrial dysfunctions (31-33). Subsequently, the mitochondrial membrane potential and expression of B-cell lymphoma-2 (Bcl-2) was decreased, while the expression of caspase-3 (CASP3) was increased, which resulted in mitochondrial apoptosis in EOC cells. Most noteworthy, we found that the migration and invasion of EOC cells were also inhibited by overexpression of CLPP or stimulation of ONC201, which may be because disorder of the mitochondrial respiratory chain induced by CLPP also caused the movement capacity of tumor cells. A lack of direct evidence to prove this was a limit of this study, although it has been reported that pro-apoptotic BCL2-associated X (Bax) promotes mesenchymalepithelial transition by binding to mitochondrial respiratory complex-I and antagonizing the malignant actions of prosurvival Bcl-2 proteins (34). The detailed mechanism of how CLPP regulates EOC cells migration requires further study.

In conclusion, altering the expression of CLPP leads to mitochondrial dysfunction and affects the proliferation and migration of EOC cells. CLPP may be a target to treat EOC.

\section{Acknowledgments}

Funding: This study was supported by funding from the Henan Provincial Department of Science and Technology Research Project (No. 172102310301).

\section{Footnote}

Reporting Checklist: The authors have completed the MDAR reporting checklist. Available at https://dx.doi. org/10.21037/atm-21-4321

Data Sharing Statement: Available at https://dx.doi. org/10.21037/atm-21-4321

Conflicts of Interest: All authors have completed the ICMJE uniform disclosure form (available at https://dx.doi. org/10.21037/atm-21-4321). The authors have no conflicts of interest to declare.

Ethical Statement: The authors are accountable for all aspects of the work in ensuring that questions related to the accuracy or integrity of any part of the work are appropriately investigated and resolved. The study protocol was reviewed and approved by the Institutional Review Board of Cancer Hospital Affiliated to Zhengzhou 
University (No. 20191016), in accordance with the Declaration of Helsinki (as revised in 2013), and informed consent was taken from all the patients.

Open Access Statement: This is an Open Access article distributed in accordance with the Creative Commons Attribution-NonCommercial-NoDerivs 4.0 International License (CC BY-NC-ND 4.0), which permits the noncommercial replication and distribution of the article with the strict proviso that no changes or edits are made and the original work is properly cited (including links to both the formal publication through the relevant DOI and the license). See: https://creativecommons.org/licenses/by-nc-nd/4.0/.

\section{References}

1. Sung H, Ferlay J, Siegel RL, et al. Global Cancer Statistics 2020: GLOBOCAN Estimates of Incidence and Mortality Worldwide for 36 Cancers in 185 Countries. CA Cancer J Clin 2021;71:209-49.

2. Lheureux S, Gourley C, Vergote I, et al. Epithelial ovarian cancer. Lancet 2019;393:1240-53.

3. Kuroki L, Guntupalli SR. Treatment of epithelial ovarian cancer. BMJ 2020;371:m3773.

4. Lheureux S, Braunstein M, Oza AM. Epithelial ovarian cancer: Evolution of management in the era of precision medicine. CA Cancer J Clin 2019;69:280-304.

5. Bowtell DD, Böhm S, Ahmed AA, et al. Rethinking ovarian cancer II: reducing mortality from high-grade serous ovarian cancer. Nat Rev Cancer 2015;15:668-79.

6. Horowitz NS, Miller A, Rungruang B, et al. Does aggressive surgery improve outcomes? Interaction between preoperative disease burden and complex surgery in patients with advanced-stage ovarian cancer: an analysis of GOG 182. J Clin Oncol 2015;33:937-43.

7. Zhang C, Li Y, Zhao W, et al. Circ-PGAM1 promotes malignant progression of epithelial ovarian cancer through regulation of the miR-542-3p/CDC5L/PEAK1 pathway. Cancer Med 2020;9:3500-21.

8. Marchetti C, De Felice F, Perniola G, et al. Role of intraperitoneal chemotherapy in ovarian cancer in the platinum-taxane-based era: A meta-analysis. Crit Rev Oncol Hematol 2019;136:64-9.

9. Christie EL, Bowtell DDL. Acquired chemotherapy resistance in ovarian cancer. Ann Oncol 2017;28:viii13-5.

10. Liu HD, Xia BR, Jin MZ, et al. Organoid of ovarian cancer: genomic analysis and drug screening. Clin Transl Oncol 2020;22:1240-51.
11. Yousefi M, Dehghani S, Nosrati R, et al. Current insights into the metastasis of epithelial ovarian cancer - hopes and hurdles. Cell Oncol (Dordr) 2020;43:515-38.

12. Lengyel E. Ovarian cancer development and metastasis. Am J Pathol 2010;177:1053-64.

13. Zong WX, Rabinowitz JD, White E. Mitochondria and Cancer. Mol Cell 2016;61:667-76.

14. Corydon TJ, Bross P, Holst HU, et al. A human homologue of Escherichia coli ClpP caseinolytic protease: recombinant expression, intracellular processing and subcellular localization. Biochem J 1998;331:309-16.

15. Goard CA, Schimmer AD. Mitochondrial matrix proteases as novel therapeutic targets in malignancy. Oncogene 2014;33:2690-9.

16. Ishizawa J, Zarabi SF, Davis RE, et al. Mitochondrial ClpP-Mediated Proteolysis Induces Selective Cancer Cell Lethality. Cancer Cell 2019;35:721-737.e9.

17. de Sagarra MR, Mayo I, Marco S, et al. Mitochondrial localization and oligomeric structure of HClpP, the human homologue of E. coli ClpP. J Mol Biol 1999;292:819-25.

18. Luo J, Zeng B, Tao C, et al. ClpP regulates breast cancer cell proliferation, invasion and apoptosis by modulating the Src/PI3K/Akt signaling pathway. PeerJ 2020;8:e8754.

19. Wong KS, Houry WA. Chemical Modulation of Human Mitochondrial ClpP: Potential Application in Cancer Therapeutics. ACS Chem Biol 2019;14:2349-60.

20. Prabhu VV, Morrow S, Rahman Kawakibi A, et al. ONC201 and imipridones: Anti-cancer compounds with clinical efficacy. Neoplasia 2020;22:725-44.

21. Guan LY, Lu Y. New developments in molecular targeted therapy of ovarian cancer. Discov Med 2018;26:219-29.

22. Cole A, Wang Z, Coyaud E, et al. Inhibition of the Mitochondrial Protease ClpP as a Therapeutic Strategy for Human Acute Myeloid Leukemia. Cancer Cell 2015;27:864-76.

23. Kotschy A, Szlavik Z, Murray J, et al. The MCL1 inhibitor S63845 is tolerable and effective in diverse cancer models. Nature 2016;538:477-82.

24. Kuntz EM, Baquero P, Michie AM, et al. Targeting mitochondrial oxidative phosphorylation eradicates therapy-resistant chronic myeloid leukemia stem cells. Nat Med 2017;23:1234-40.

25. Konopleva M, Pollyea DA, Potluri J, et al. Efficacy and Biological Correlates of Response in a Phase II Study of Venetoclax Monotherapy in Patients with Acute Myelogenous Leukemia. Cancer Discov 2016;6:1106-17.

26. Gispert S, Parganlija D, Klinkenberg M, et al. Loss of mitochondrial peptidase Clpp leads to infertility, hearing 
loss plus growth retardation via accumulation of CLPX, mtDNA and inflammatory factors. Hum Mol Genet 2013;22:4871-87.

27. Ishizawa J, Kojima K, Chachad D, et al. ATF4 induction through an atypical integrated stress response to ONC201 triggers p53-independent apoptosis in hematological malignancies. Sci Signal 2016;9:ra17.

28. Arrillaga-Romany I, Chi AS, Allen JE, et al. A phase 2 study of the first imipridone ONC201, a selective DRD2 antagonist for oncology, administered every three weeks in recurrent glioblastoma. Oncotarget 2017;8:79298-304.

29. Stein MN, Bertino JR, Kaufman HL, et al. First-inHuman Clinical Trial of Oral ONC201 in Patients with Refractory Solid Tumors. Clin Cancer Res 2017;23:4163-9.

30. Kline CL, Van den Heuvel AP, Allen JE, et al. ONC201 kills solid tumor cells by triggering an integrated stress response dependent on ATF4 activation by specific eIF $2 \alpha$ kinases. Sci Signal 2016;9:ra18.

Cite this article as: Kou X, Ding H, Li L, Chao H. Caseinolytic protease P (CLPP) activated by ONC201 inhibits proliferation and promotes apoptosis in human epithelial ovarian cancer cells by inducing mitochondrial dysfunction. Ann Transl Med 2021;9(18):1463. doi: 10.21037/atm-21-4321
31. Rak M, Rustin P. Supernumerary subunits NDUFA3, NDUFA5 and NDUFA12 are required for the formation of the extramembrane arm of human mitochondrial complex I. FEBS Lett 2014;588:1832-8.

32. Renkema GH, Wortmann SB, Smeets RJ, et al. SDHA mutations causing a multisystem mitochondrial disease: novel mutations and genetic overlap with hereditary tumors. Eur J Hum Genet 2015;23:202-9.

33. Lau E, Freitas P, Costa J, et al. Loss of mitochondrial SDHB expression: what is its role in diffuse thyroid lipomatosis? Horm Metab Res 2015;47:165-7.

34. Kim EM, Jung CH, Song JY, et al. Pro-apoptotic Bax promotes mesenchymal-epithelial transition by binding to respiratory complex-I and antagonizing the malignant actions of pro-survival Bcl-2 proteins. Cancer Lett 2018;424:127-35.

(English Language Editor: B. Draper) 